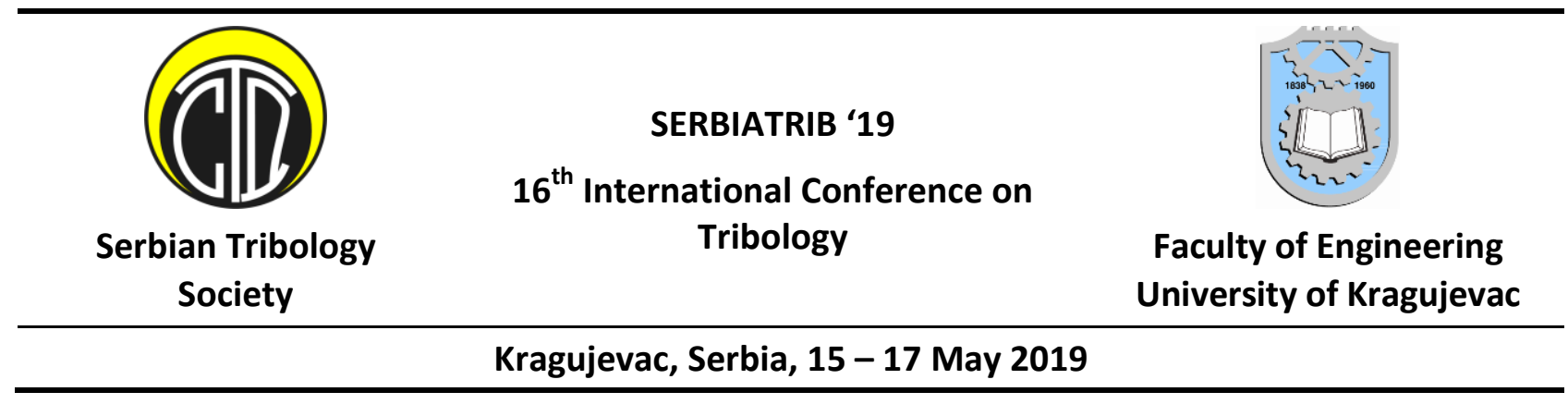

\title{
FRICTION MODELING IN SIMULATING BALLISTIC IMPACT. A REVIEW
}

\author{
George Ghiocel OJOC ${ }^{1,2}$, Catalin PIRVU ${ }^{3}$, Simona BADEA ${ }^{4}$, Lorena DELEANU ${ }^{1}$ \\ 1"Dunarea de Jos" University of Galati, Galati, Romania \\ ${ }^{2}$ Maritime University Constanta, Romania \\ ${ }^{3}$ INCAS - National Institute for Aerospace Research "Elie Carafoli", Bucharest, Romania \\ ${ }^{4}$ Scientific Research Center for CBRN Defense and Ecology, Bucharest, Romania \\ *Corresponding author: lorena.deleanu@ugal.ro
}

\begin{abstract}
This paper presents a review on using friction coefficient in modelling ballistic impact. The are discussed on friction introduced in modelling the impact at different levels: micro (fiber and yarns), meso (woven or unidirectional fabrics) and macro (layered or equivalent monoblock structures). Recent works simulating the impact projectile - target, done at different levels (micro, meso, macro) pointed out the influence of friction coefficient between bodies in contact. In all cited references, the friction coefficient is kept constant, even if experimental studies proved that it has different values for different sliding speeds, especially for contact metal - polymer as in the impact of aramid fabric armor.
\end{abstract}

Keywords: friction coefficient, impact velocity.

\section{INTRODUCTION}

Myshkin et al [1] discussed the influence of load and velocity on the friction coefficient and graphically resumed (Fig. 1) the research studies of [2], [3], [4], [5], but for low velocity, from $0.1 \mathrm{~m} / \mathrm{s}$ to $2 \mathrm{~m} / \mathrm{s}$, values that are far bellow the values charactering the impact velocity of projectiles $(100 \ldots 1000 \mathrm{~m} / \mathrm{s})$. Also, the load influences the value of friction coefficient of the same couple of materials in dry conditions.

When the projectile is arrested by the target, the friction coefficient is not constant, having values for high, medium and low velocities, also depending on the temperature between the moving contact surfaces. Also, high friction occurs when projectile is deformed on or into the target.

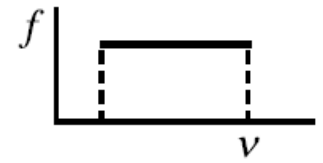

steel-polymer $10^{-4} \ldots 10^{-2} \mathrm{~m} / \mathrm{s}[2]$

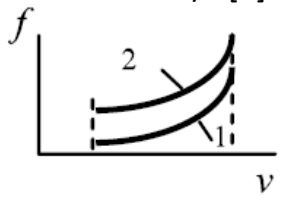

$10^{-3} \ldots 10^{-1} \mathrm{~m} / \mathrm{s}$ steel-PTFE (1), steel-PA (2) [4]

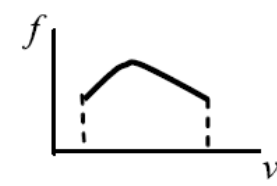

$0.04 \ldots 1.8 \mathrm{~m} / \mathrm{s}$

[3]

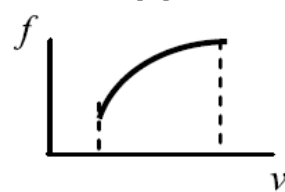

$1.5 \times 10^{-2} \mathrm{~m} / \mathrm{s}$, steel -PTFE, rubber

[5], [6]
Figure 1. Variants of the dependence of friction coefficient on sliding velocity, Steel - polymer 
Many engineering designs are based on considering friction coefficient as a constant of materials in contact, thus, independent of velocity, load, temperature, size of contact. This is acceptable as long as the tribolayers do not change their mechanical and thermal characteristics. It is difficult to impossible to practically separate the effect of these factors. Also, the impact is characterized by very dramatic failure processes, overlapping on friction and measuring the energy dissipated by friction alone is still quite an adventure.

Sliding friction steel-polymer may be discussed taking into account the mechanical an thermal influences:

- at low velocity, thermal effect is reduced but the effect of adhesion and viscous resistance, especially under higher load, could make the value of friction coefficient higher and with big oscillations,

- at high velocity, elastic characteristics are more influent and the value of friction coefficient will depends only slightly on velocity or it will decrease with the increasing velocity; frequently, the application involving high velocity contact last less time and thermal effect could be neglectable,

- for medium velocity between bodies in contact, visco-elastic and thermal effects are present, most of the polymers sliding on steel having a maximum value of the friction coefficient.

This qualitative analysis is difficult to put into practice, that is limiting these three domains with actual values for velocity, even at laboratory scale.

Stachowiak [7] mentioned the exerimental work of Ettles [8] in 1987 for pointing out that polymers like LPDE, PP and PA had a maximum value for friction coefficient in the range of $0.3 \ldots 8 \mathrm{~m} / \mathrm{s}$ (close to 1 or even above 1 ), after that this value decreasing dramatically, but tests were done under $100 \mathrm{~m} / \mathrm{s}$, value that is at the lower level for ballistic impact. Another study done by Bueche and Flom [9] in 1958 for steel sliding against unlubricated plexiglass at different temperatures reveals that above 5 $\mathrm{m} / \mathrm{s}$ the friction coefficient is not influenced by the temperature.

\section{FACTORS AFFECTING FRICTION WHEN A PROJECTILE HITS A TARGET}

Based on the principal of conservation of energy and the relation given by Nilakantan et al. [10 Nilakantan, 2015] for the energy transformations that occur during the projectile-fabric impact, the follwoing energy balance may be written:

$$
\begin{gathered}
E_{\text {projectile }}^{\text {initial }}=E_{\text {projectile }}^{\text {residual }}+E_{\text {fabric }}^{\text {internal }}+E_{\text {fabric }}^{\text {kinetic }} \\
+E_{\text {fabric }}^{\text {friction }}+E_{\text {projectil }}^{\text {deformation }}+ \\
+E_{\text {projectil-fabric }}^{\text {friction }}+E_{\text {fabric }}^{\text {miscellanous }}
\end{gathered}
$$

The loss of projectile kinetic energy is equal to the summation of the three main fabric energy dissipating mechanisms:

- fabric internal energy due to tensile yarn elongation,

- fabric kinetic energy due to projectilefabric momentum transfer, and

- frictional energy due to inter-filament, inter-yarn, and projectile-fabric frictional sliding interactions.

Internal energy due to other deformations such as yarn transverse compression and yarn shearing are usually much smaller than that due to tensile straining especially for anisotropic fibers, such as aramid. Other miscellaneous energy losses, such as acoustic and heat, are relatively very small in some application, including for individual armors, and can be neglected, but for heavy threats should be taken into account

The factors affecting friction in impact process include:

- materials of the bodies in impact (projectile, target), by their mechanical and thermal characteristics,

- size and structure of target (metallic, fibre, ceramics)

- size and shape of the projectile

velocity and mass of the projectile

Thus, friction should be analyzed for particular cases of impact.

During the projectile impact with the target, friction process could have different aspects:

- friction between projectile and target without penetration (the projectile is 
rebonded); friction is initiated in a layered target, but with small displacements, reducing the influence of friction in energy dissipation,

- friction between the projectile and the broken layers and the consequently friction between layers that are laterally tensioned (delamination with friction after).

For the similar projectile, the target could be designed against penetration using different materials and even combinations of them.

Protection systems could made of steel (monoblock or sheets), fabrics manufactured from woven or unidirectional polymeric fibres gained the interest in protecting individuals, usually in layers, also ceramic plate are introduced rarely alone, often in addition to first ones [11]. When impacting the target, friction depends on materials in contact and, especially for fibres, on their arrangement (2D and 3D woven, unidirectional etc.) and coating, if the structure is layered or monoblock (for transparent windows) etc.

Introduction of friction in simulation of impacts has to be done for the particular case of interest.

In simulations, friction was introduced for micro, meso and macro level.

Sockalingam [12] published a review analyzing all these levels in order to finally discussed the complex multi-scale system of fabrics, woven or not, taking into account the structural hierarchy of the materials, anisotropic material behavior, projectile-fabric interactions, impact velocity and boundary conditions and also the friction between contacting bodies.

Frictional mechanisms, including fiber breakage at the yarn cross-overs, flattening and rupture of fibers and yarn pull out, are also physically observed.40

Unlike aramid fibers, the melting temperature of PE is low $\left(110 \ldots 145{ }^{\circ} \mathrm{C}\right)$. Prevorsek et al. [13] reported that the projectile-fiber frictional interactions result in an increase in temperature on the projectiletarget interface, above the melting point of this PE fiber. Due to the short time of ballistic impact, the temperature rise is limited to a small region and its effects on the target performance are not significant, even if, locally, the polymer is soften to molten.

\section{FRICTION BETWEEN FIBERS}

The micro level simulation of the impact implies model for a fiber or a yarn.

The apparent friction (defined as the ratio of lateral scratch force to normal indentation force) is reported to increase up to $300 \%$ higher than the aramid yarn-yarn friction of 0.2-0.3. Apparent fiber-fiber and yarn-yarn friction are an important energy dissipative mechanisms due to increased apparent friction associated with these fibres [14].

Sockalingam et al. [15] simulated the behavior of a fiber under impact taking into account a friction coefficient of 0.2 , mesh size of $1.8 \mu \mathrm{m}$, fiber length $500 \mu \mathrm{mm}$, but introducing friction, the tensile stress near the contact with the projectile may increase, even if impact energy is now dissipated also by friction.

The fiber friction with the contacting bodies (projectile and other fibres) and mechanical and thermal properties play a substantial role in slowing down the projectile, bullet deformation and arrest. The friction coefficient of aramid fibers is higher as compared to UHMWPE fibers, which are slicky, highly oriented, high strength and exibit lower friction. The low friction in fabrics, in layered arrangement, is overcome by adding higherfriction coatings (polymeric foil of PE or other thermoplastic polymers, having the thickness of microns) to the ballistic fabric surface. Also, more rigid matrix for fabrics [16] (like polyvinyl butyral or other similar for helmets) improve the ballistic resistance.

One factor that influences the ballistic resistance is the friction between fibers during projectile penetration. Controlled friction between fibers is desirable to slow down and deform the projectile. If friction between fibers is too high, one fiber will make the other fiber to fail during projectile penetration and reduces the resistance of the whole protection system. If fiber-to-fiber friction is too low, the material will not offer any resistance. 


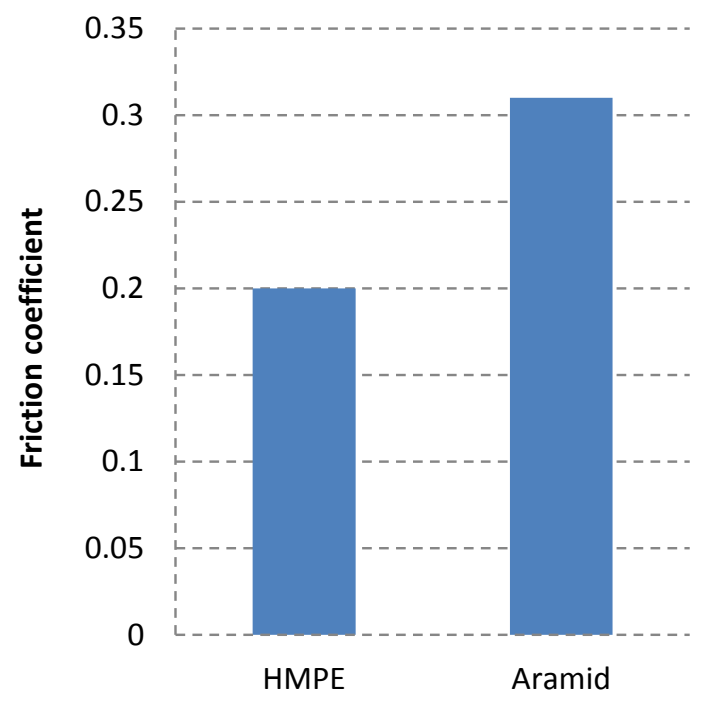

Figure 2. Coefficient of friction for yarn-to-yarn moving contact [17]

In fabrics, friction is increased by changing the fiber orientation (for instance, fabrics are composed by two, three or four layers, with unidirectional fibers, arranged at relative angles $\left.\left[0^{\circ}, 90^{\circ}, 45^{\circ},-45^{\circ}\right]\right)$, by a coating at fiber level or fabric level, by bonding very thin foils on the fabrics (sometimes on each layer). Quilting/sewing with yarns of lower properties, at regular intervals of the unidirectional yarns, increases fiber-to-fiber friction, as they maintain the fibers and the yarns in contact.

The frictional properties of projectile-tofiber interaction may be modified by adding a suitable polymer coating, during the manufacturing of the protection system. Both woven and non-woven aramid and HDPE plied materials have an increased ballistic resistance for a certain number of threats. If the coating is not adequately selected, it will increase the weight and stiffness of ballistic material without improving its ballistic resistance of individual armor [17].

When the projectile is passing through layer after layer, more and more fibers become engaged with the bullet and increase the friction, sufficient enough to slow it down, especially when the bullet is strongly deforming.

Rebouillat et al. [18] investigated the tribological properties of the Kevlar 29 woven fabric and its yarns. They measured the friction between several Kevlar 29 fabrics and between their Kevlar 29 yarns, with different surface treatments, under various sliding speeds and the inter-yarn friction coefficients was in the ranged of 0.2 and 0.4 . No significant change of the inter-yarn friction coefficient occurred as the sliding velocity increased from $96 \mathrm{~mm} / \mathrm{min}$ to $600 \mathrm{~mm} / \mathrm{min}$, but friction varied significantly as the sliding speed increased.

Each type of high performance ballistic fiber have particular characteristics. Aramid fibers have higher fiber-to-fiber friction than HMPE fibers (Fig. 2), making the bullet outer jacket to crack easier. HMPE fibers have non-linear viscoelastic properties, which help to arrest bullet fragments better than aramid fibers (that could be consider linear when compared). Using layers of high friction material at the front and capturing fragmented bullets by HMPE offers a lighter weight solution to stop the bullet at a lower weight than either a 100\% aramid or $100 \%$ HMPE fiber individual armor [17]. The effect of increasing friction by coating or combining different types of fibers is far from being explained as there is a synergic response of the new designed protection system and simulation and tests could reveal influences far from being linear or added one to another (Fig.3).

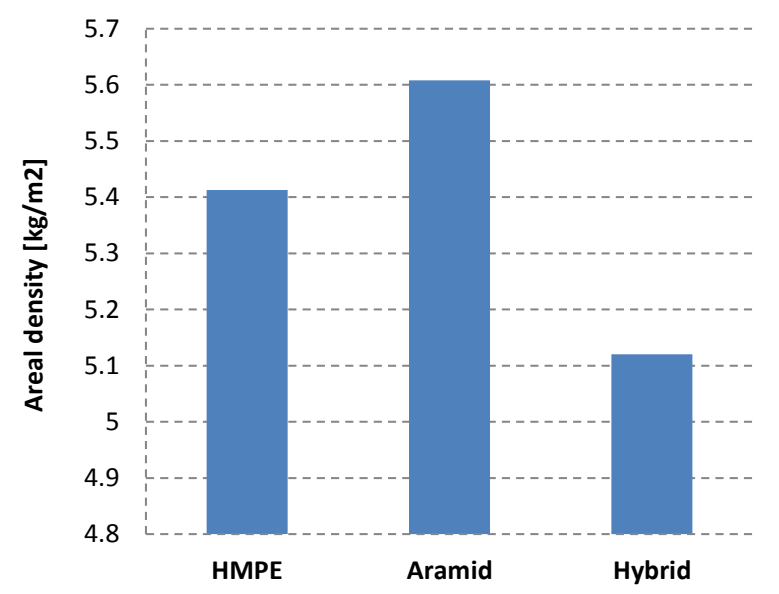

Figure 3. Effect of hybrid protection system for an individual armor level NIJ IIIA [17]

For protection systems made of fabrics, the frictional energy dissipated during the impact is a non-linear energy absorption mechanism. Friction mechanisms include friction due to slippage of yarns, interaction of adjacent layers and interaction projectile-target. Many factors influence the amount of energy dissipated by friction, including the contacting 
yarns, armor boundary conditions allowing or restricting yarn motion. Bhatnagar [17] concluded that, at lower impact velocities, elongation, abrasion and fibrillation of fibers are more important in arresting the projectile.

The energy absorbed during the impact is mainly due to the compression of the yarns around the projectile and the dissipated energy due to the friction between the yarns [19]. The presence of this type of perforation versus shear plugging in dry fabrics and laminates is highly affected by the projectile nose shape [20].

The final mechanism, common to all projectile types and most material systems is friction and is simply the energy required to push the projectile through the crater created by either hole expansion or plugging. The frictional load is related to the length of penetrator in contact with the panel, the inplane compressive stresses acting on the penetrator and the coefficient of friction between penetrator and composite [17].

\section{Friction in Fabrics}

Mechanical properties of a fabric are different from the constitutive yarns, due to its complex arrangement. Yarn crimp, friction and yarns' interaction change the response of a fabric to a particular threat. Cunniff [21] discussed the loss of efficiency in going from a fibre to a yarn, from a yarn to a fabric, and from a single fabric layer to multi-layer packs. He concluded that yarn slippage may lead to the loss of efficiency and performance degradation in a loosely woven fabric or a fabric with low yarn-to-yarn friction.

Considering the geometry of the weave, balanced fabrics (plaine, for instance) absorb more energy than non-balanced ones (twill or satin), but o more dens weaving induce a process of yarn degradation, the bending induce by the weave reducing the mechanical characteristics of yarns.

In studies published by Lee et al. [22] and Rudov-Clark et al. [23] degradation of glass yarn properties during the weaving process of three-dimensional fabrics is discussed. The weaving damage mainly influences the yarn strength, reducing it by up to $30 \%$ due to the high abrasion of the filaments. The tensile modulus of the yarns was found to be less affected by weaving.

Lim et al. [24] developed a finite-element model of ballistic impact on Twaron fabric. A non-linear, explicit, 3D finite-element was used for simulating the behavior of the fabric under high-velocity impact. The fabric is modeled using membrane elements. Suitable material properties to account for its viscoelastic nature are obtained through mathematical manipulation of the threeelement spring-dashpot model and by using available experimental data. The ballistic limit, residual velocity, energy absorption and transverse deflection profiles of the fabric are predicted and compared to those from tests.

Lim et al. [19] studied two-ply fabrics impacted by the same projectile geometries. They concluded that while target performance is highly affected by the projectile nose-shape, the influence diminishes in the thicker panels. They also observed that while failure throughrupture and friction is more evident on the impact face, bowing is more amplified on the back-face of the target (Fig. 4).
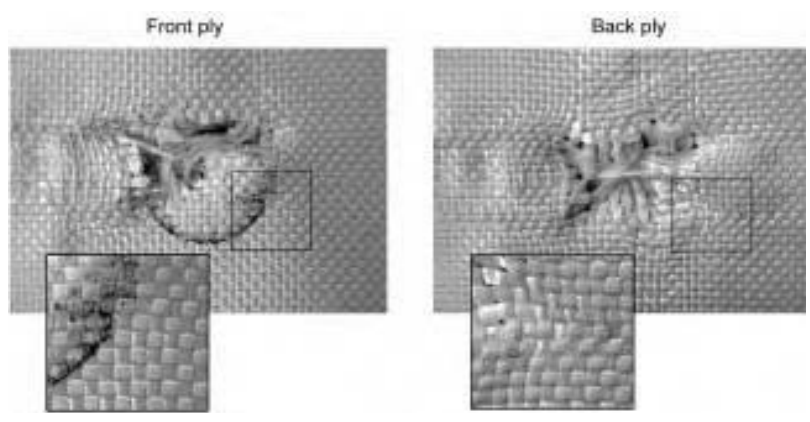

Figure 4. Increase in bowing of the yarns on the backside of the target [19]

Recent studies [19], [24] have included the effect of transverse yarn interactions and have found that these interactions can significantly influence the results from ballistic response models. The description of single ply fabric deformation is given to serve as an illustrative example to point out some of the fundamental physical mechanisms observed that influence the ballistic performance of fabrics. Material properties, fabric structure, projectile 
geometry, impact velocity, multiple ply interaction, far field boundary conditions and friction all play a role. Although many authors attempt to describe these mechanisms individually, it should be noted that many of the individual mechanisms have been reported in a coupled manner (i.e. multiple ply ballistic panels impacted by different geometry projectiles at varying velocities). As such, it is difficult to isolate each mechanism; therefore, further research in this aspect is needed.

The failure modes of aramid fibers are splitting and fibrillation, but also shearing, thinning (Fig. 5, [25]). PBO fiber also shows similar behavior as the aramid one. Nylon-66 fiber exhibits melting. The UHMWPE fiber, such as Spectra, exhibits straining, kinking due to the strain, as well as snap-back of fibers after breakage. The evidence of melting was also observed for Spectra fibers. To explain this phenomenon, the following two opposite arguments were reported: a) the melting is due to the heat generated from the targetprojectile friction, during the penetration, b) adiabatic heating effect after the penetration.

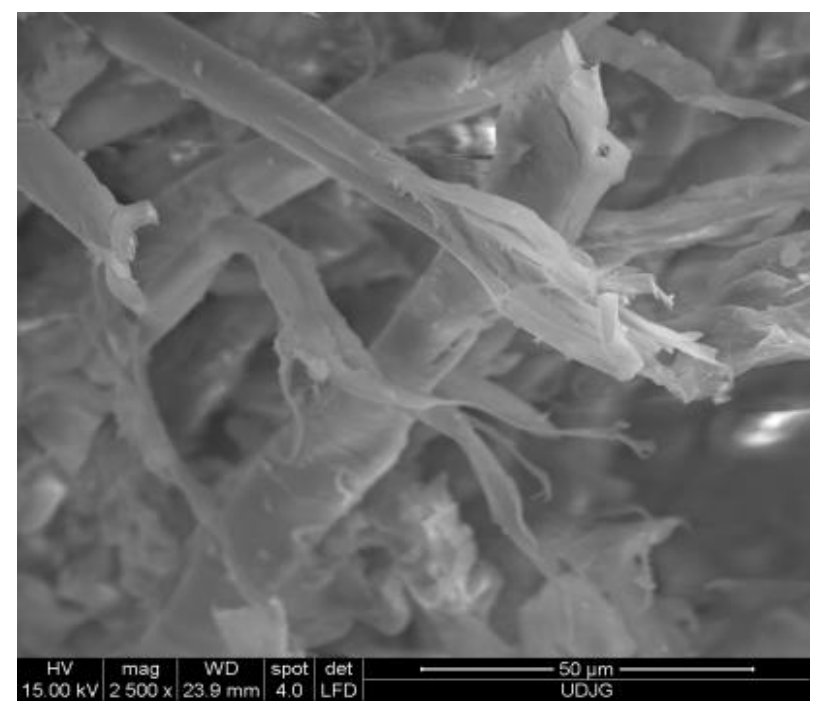

Figure 5. Typical failures of aramid fibers, after a ballistic impact with a 9mm FMJ on 24 layers of fabric SB1, bonden with PVB [25], [26]

In ballistic resistant structures, filament yarns are used to absorb projectile impact force. The logic behind the use of filaments is to present a network of high modulus, high strength fiber structure components that individually extend the entire breadth or length of the structure into which a ballistic impact is directed.
Sockalingam et al. [27] modeled the yarn as a system of fibres, including the effect of intrayarn friction The sensitivity of friction between the fibers is studied by choosing a small and a high value of the coefficient of friction and the predicted force displacement curves are shown in Fig. $6 \mathrm{~b}$.

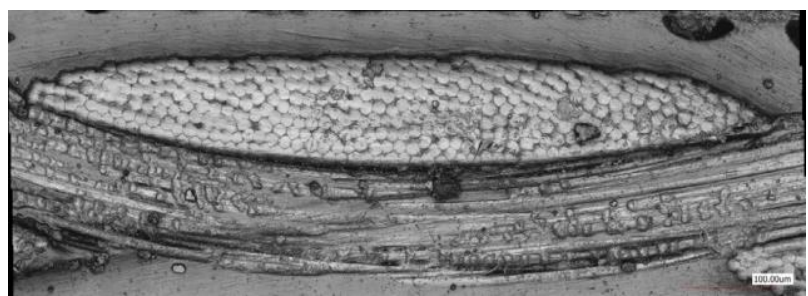

a) Actual yarn formed by aramid fibers (unloaded)

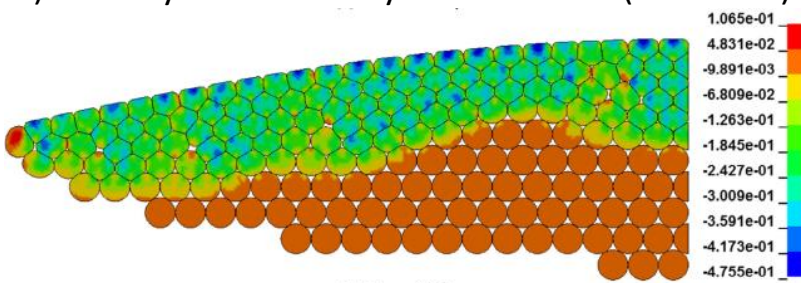

b) Contours of compressive strains at $324 \mathrm{~m} / \mathrm{s}$ impact at the moment $7 \times 10-5 \mathrm{~s}$

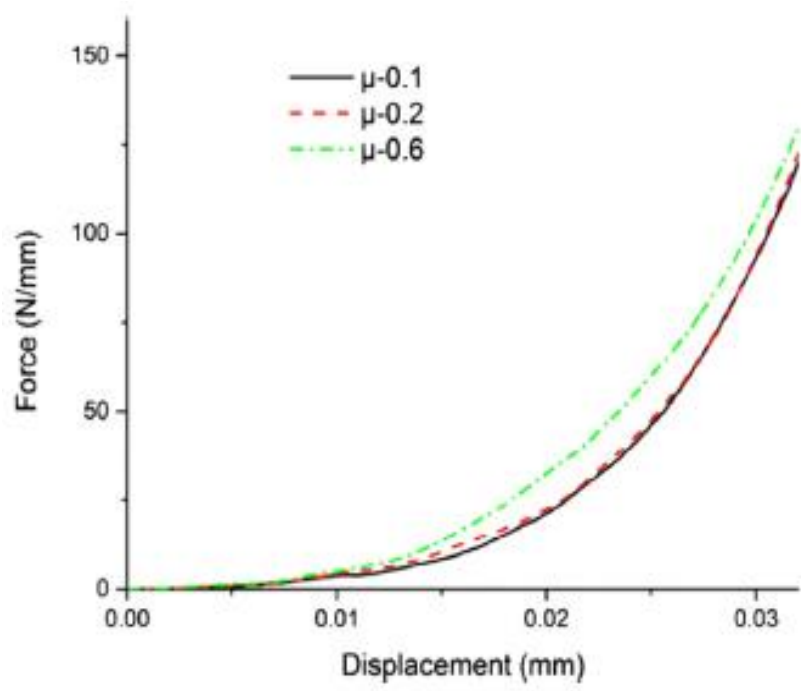

c) Influence of friction coefficient value

Figure 6. Tow transverse compression response of Kevlar KM2 fibers, using an anisotropic constitutive model [27]

The numerical predictions indicate there may be a dependence on the friction coefficient in the finite strain regime with a higher force required to compress the fibers with larger friction coefficient (Fig. 6c).

Among findings during this development, it was clear that significant advantage exists where HPPE/ECPE fibers are 5.5 denier or finer. Disadvantage was observed when fiber blends with PBO present were tested because 
of the very low frictional characteristics of these fibers [28].

Duan et al. [29] developed a finite-element model to study the influence of friction during ballistic impact of a rigid sphere on a square woven fabric that was firmly fixed along its four edges. Projectile-fabric friction and yarnyarn friction were investigated and from the modeling result indicates that friction dramatically affects the local fabric structure at the impact region by hindering the lateral mobility of principal yarns. Reduction of lateral yarn mobility allows the projectile to load and break more yarns so that fabric possessing a high level of friction absorbs more energy than fabric with no friction. The projectile-fabric friction delays yarn breakage by distributing the maximum stress along the periphery of the projectile-fabric contact zone. The delay of yarn breakage substantially increases the fabric energy absorption during the later stages of the impact. The yarn-yarn friction hinders the relative motion between yarns and, thus, resists de-crimping of fabric weave tightness. It induces the fabric to fail earlier during the impact process. The overall influence of projectile-fabric friction and yarnyarn friction cannot be calculated by simply superposing their individual effects. They reported a similar contribution of projectiletarget friction in their research on low velocity impacts on disks made of polymers.

\section{Modeling Fabrics under impact}

Wang et al. [30] investigated the effect of the inter-fiber friction coefficient on fabric ballistic performance (Fig. 7). Firstly, dynamic stress responses within a fabric due to first fiber failure are analyzed. Relations between interfiber friction and progressive fiber failure are assessed. Then, ballistic perforation processes of Kevlar KM2 fabrics are simulated using a fiber (micro) level. In this model, each yarn is discretized into many fibers and each fiber is divided into many rod elements. Relations of ballistic limits and inter-fiber friction coefficients are presented. In these simulations the ballistic limit improves as the inter-fiber friction coefficient increases up to a critical value. Beyond that point, ballistic strength decreases slightly as the inter-fiber friction coefficient increases. The inter-fiber friction also changes the ballistic perforation mechanisms. The effect of the friction behavior of fibers and yarns is twofold in an opposite manner. Inter-fiber friction reduces yarn mobility and reduces the outward movement of principal yarns from the impact center. As a result, more yarns participate in resisting the projectile, which improves the ballistic performance of the fabric. On the other hand, inter-fiber friction restricts the relative motion between fibers that, in turn, generates a higher fiber stress wave at the vicinity of a failed fiber element. Consequently, a fabric with a higher interfiber friction coefficient could be more susceptible to unstable damage propagation, which may not be beneficial to its ballistic strength

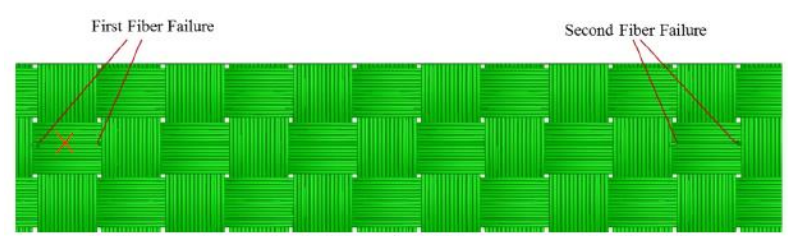

Figure 7. the model proposed by [30]

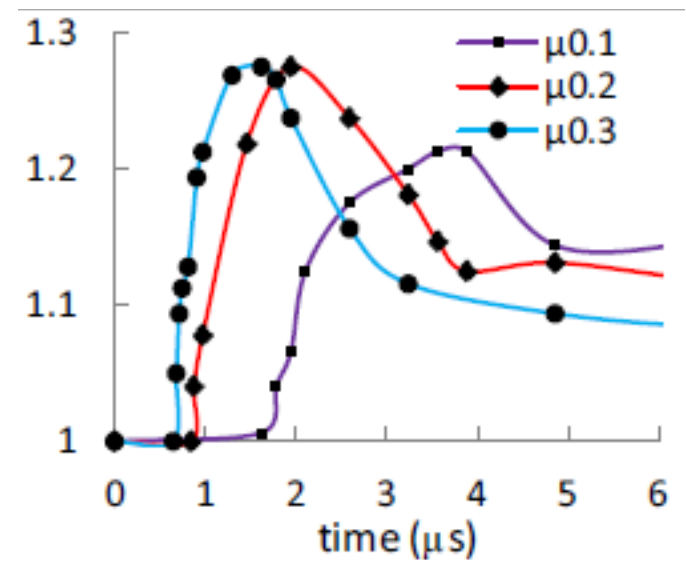

Figure 8. Stress concentration ratio depending on friction coeffient value [30]

Inter-fiber friction coefficients of 0.3 or greater are also applied in the numerical simulations. Results indicate employment of a larger friction coefficient causes fiber damage to propagate not only in the longitudinal direction, but also in the transverse direction. The damage mechanism is explained as 
follows: As a fiber element breaks, a portion of the load originally supported by the broken fiber is transferred to its neighboring fibers through inter-fiber friction, generating stress concentration (overload) in the neighboring fibers (Fig. 8). If the stress of a neighboring fiber is greater than its fiber strength, the neighboring fiber will fail, resulting in transfiber failure propagation. This could further propagate into the surrounding neighboring fibers and trigger trans-fiber failure in a yarn. Cracks in the fabric demonstrate the consequence of this progressive trans-fiber damage propagation. Numerical results indicate trans-fiber damage propagation is more pronounced in fabrics with increased inter-fiber friction.

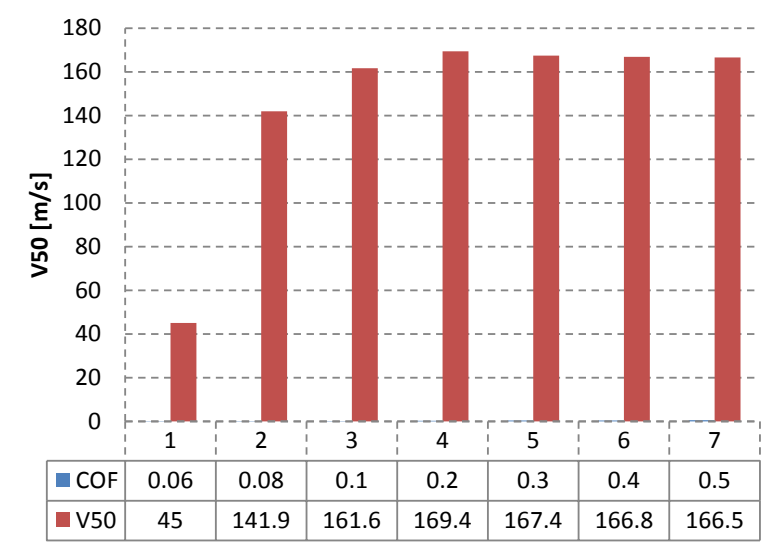

Figure 9. Effect of friction coefficient on V50 in simulations on the $V_{50}$, the experimental value being $V_{50 \text { experimental }}=132.9 \mathrm{~m} / \mathrm{s}$

Table 1. Numbers of failed yarns after impact

\begin{tabular}{|l|l|l|l|l|}
\hline Friction coefficient & 0.06 & 0.1 & 0.3 & 0.5 \\
\hline Number of failed wefts & 0 & 2 & 4 & 6 \\
\hline Number of failed warps & 0 & 2 & 4 & 6 \\
\hline
\end{tabular}

Duan [31] derived from numerical simulations, the ballistic resistances of fabrics at various interfiber friction levels (Fig.9). In order to examine the role of friction during ballistic impact of high-strength fabric structures, a model the ballistic impact of a rigid sphere into a square patch of plain-weave fabric. Two types of boundary conditions were applied on the fabric: four edges clamped and two opposite edges clamped. Simple Coulomb friction was introduced between yarns at crossovers and between the projectile and the fabric. Results show that the friction contributed to delaying fabric failure and increasing impact load (Fig. 10). The delay of fabric failure and increase of impact load allowed the fabric for absorbing more energy. Fabric boundary condition is a factor that influenced the effect of friction. The fabric more effectively reduced the projectile residual velocity when only two edges were clamped.
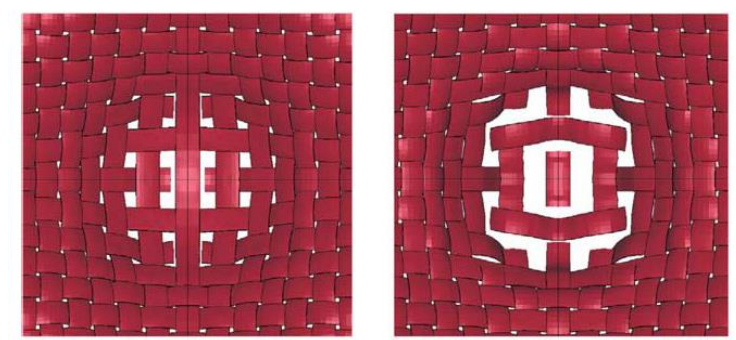

$\mu=0$
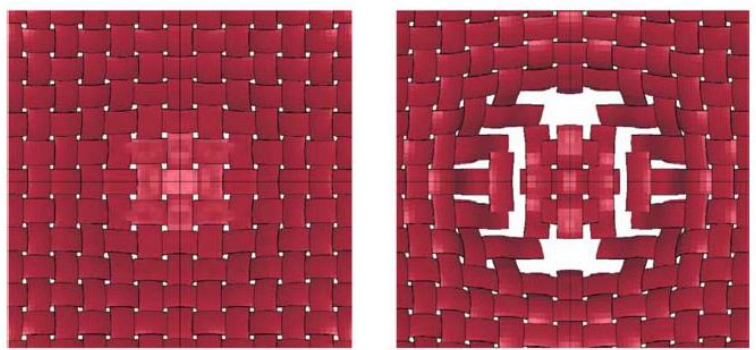

$\mu=0.5$

$4 \mu \mathrm{s}$

$5 \mu \mathrm{s}$

Figure 10. The impacted region of the fabric, at two moments, all edges fixed [31]

Only impact velocity and residual velocity of the projectile are measured in ballistic impact experiments. The role of friction during an impact process, is hard to resolve through experimentation only. This is because it is very difficult or even impossible to obtain detailed information on fabric deformation and failure. For a better understanding of the ballistic impact of fabric structures, analytical or numerical models are necessary. The role of friction during the ballistic impact is explored by comparing the fabric deformation, impact load, and energy absorption capacity at different friction conditions, the failed yarns (Table 1 ).

A case was modeled where a friction coefficient $\mu=0.5$ was used for both the yarnyarn friction and the projectile-fabric friction. 
Figure 11 presents the fabric deformation at different moments. As the impact process continued, the transverse deflection of the principal yarns (those yarns in direct contact with the projectile) propagated away from the impact region. The interaction between yarns at crossovers caused the secondary yarns (those yarns not directly in contact with the projectile, but having been twisted or/and displaced) to deflect out of the fabric plane. This caused the transverse deflection to become pyramidal in shape. The transverse deflection wave front formed a square with its four corners located at the two cross principal yarns that passed through the impact center. At $6 \mu \mathrm{s}$, the transverse deflection wave front did not reach the fixed edges, but the fabric had been broken at the impact region. Eventually, the projectile perforated the fabric and moved away at a constant velocity; the fabric sprung back and, at $8 \mu \mathrm{s}$, the transverse deflection became conical in shape as indicated by its largely circular base.
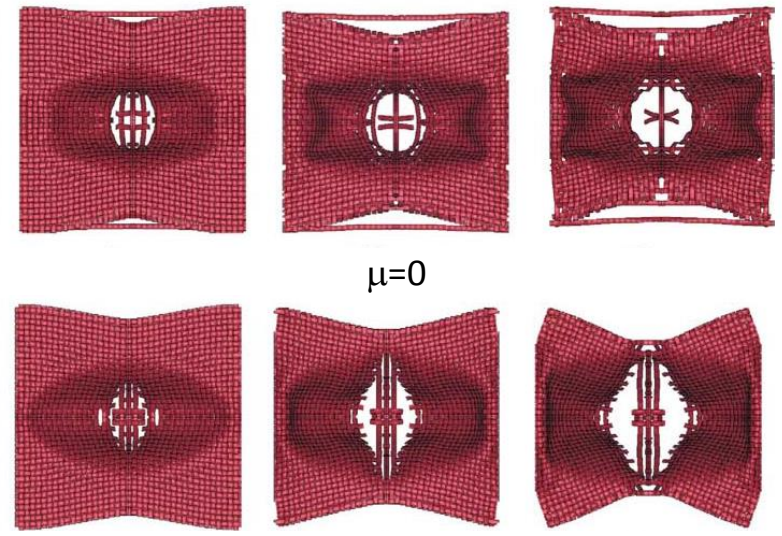

$\mu=0$

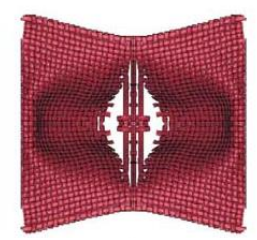

$\mu=0.5$

$8 \mu \mathrm{s}$

$12 \mu \mathrm{s}$

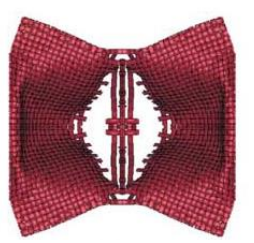

$16 \mu \mathrm{s}$
Figure 11. Different moments for two cases: without friction $(\mu=0)$ and with friction $(\mu=0.5)$

Wilde et al. [32] observed similar pyramidal- and conical- shape deformations during their photographic investigation of high-speed missile impact on a nylon fabric. Pirvu also reported piramidal shape during a test with $9 \mathrm{~mm}$ FMJ, with impact velocity of $400 \mathrm{~m} / \mathrm{s}$ (Fig. 12).

The boundary conditions significantly affected the fabric deformation. With the same friction condition, the fabric with four edges clamped slowed down the projectile more quickly than the fabric with two edges clamped; however, the fabric with two edges clamped more effectively reduced the residual velocity of the projectile.

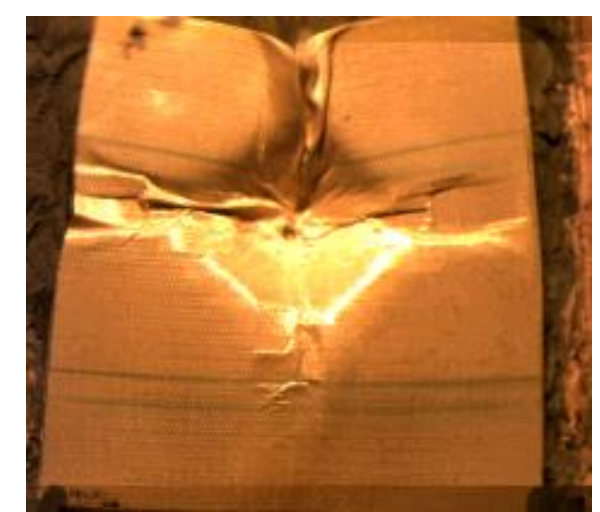

Figure 12. layers of LFT SB1, 9 mm FMJ, 400 $\mathrm{m} / \mathrm{s}$, fast camera image [26]

This is because the time needed for the projectile to perforate the fabric was much less if four edges of the fabric were clamped. with the same friction condition, the fabric energy absorption capacity was much higher if only two edges of the fabric were clamped. With the same boundary conditions, the fabric energy absorption capacity was higher when consider $\mu=0.5$. The friction effect was different at different boundary conditions; the friction increased the fabric energy absorption capacity by $11 \%$ when four edges of the fabric were clamped, whereas it increased the fabric energy absorption capacity by $24 \%$ when two edges of the fabric were clamped. The result suggests that fabric boundary condition is a factor that influenced the friction effect.

Wang [30] concluded that the ineffective zone length after first fiber breakage is related to the inter-fiber friction coefficient, for the simulation with impact velocity in the range 63 $\mathrm{m} / \mathrm{s}$...142 $\mathrm{m} / \mathrm{s}$, with spherical projectile. A lower friction coefficient yields a larger ineffective zone. A higher friction coefficient results in a higher stress concentration ratio. Fragment length after a progressive fiber failure is inverse proportional to the inter-fiber friction coefficient. Trans-fiber damage propagation occurs when the inter-fiber friction is high, which generates yarn failure or fabric cracks. In simulations, V50 increases with an increase of the friction coefficient until 
a critical value is reached. Beyond this point, V50 is insensitive to friction coefficients and decreases slightly as the friction coefficient continues to increase. Fabric damage starts from fiber cut and fibrilations along the principal yarns. As impact proceeds, high fiber stress in the impact area develops and more and more fibers fail. Perforation follows. If the inter-fiber friction coefficient is smaller than the critical inter-fiber friction coefficient, perforation is primarily due to fiber fragmentation propagating along the principal yarns. If the friction coefficient is greater than the critical friction coefficient, trans-fiber and trans-yarn damage propagates in the transverse direction. This induces more yarn failure in the perforation process. Numerical results are approximately $25 \%$ higher than experimental results.

lonescu et al. [33] did simulations with isotropic layers, having the mechanical properties of aramid yarns on longitudinal axis. The virtual residual velocities was obtained for a range of impact velocities of $150 \ldots . .400 \mathrm{~m} / \mathrm{s}$ (Fig. 13). The model run without friction has produced the highest value of the top head bullet velocity after impact for each case. The friction becomes important in energy balance of the impact when the layers are broken. When the projectile velocity is not enough for destroying the layers (at least one), the projectile is rebound, the influence of friction being noticed on a reduced time interval. These cases suggest that friction increases $V_{50}$, with $10 . . .40 \mathrm{~m} / \mathrm{s}$, at least for the studied range of initial velocity of the bullet and the bullet residual velocity is lowered.

Figure 14 [33] revealed the different behavior of the bullet and stratified panel when using different values for the friction coefficient between layers.

The selection of friction coefficient values seems to be realistic, especially for bullet-layer $\operatorname{COF}(b-I)=0.3$ and for friction between two layers $\operatorname{COF}(\mathrm{I}-\mathrm{I}) \quad 0.2 \ldots 0.4$. Images from simulations (Fig. 14) could be compared to the experimental ones and parameters that better described the layer failure could be used for future more complex simulation.
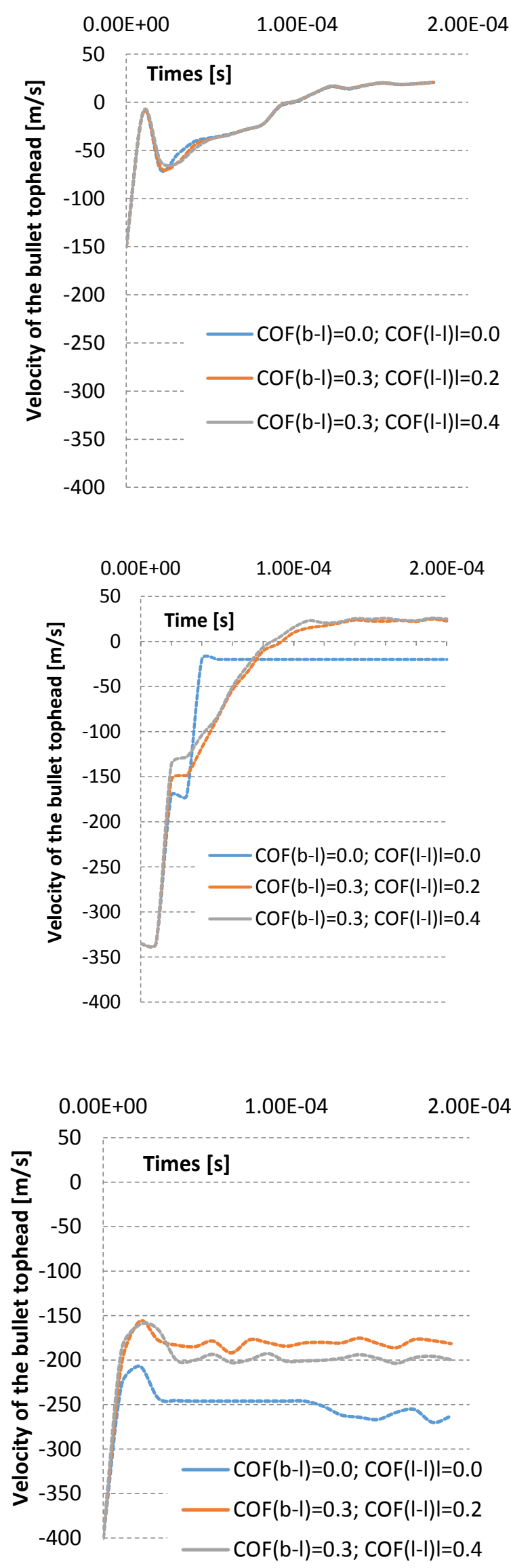

Figure 13. Macro model of the 4 layers made of virtual material with isotropic characteristics and values close to longitudinal ones of aramid yarns 


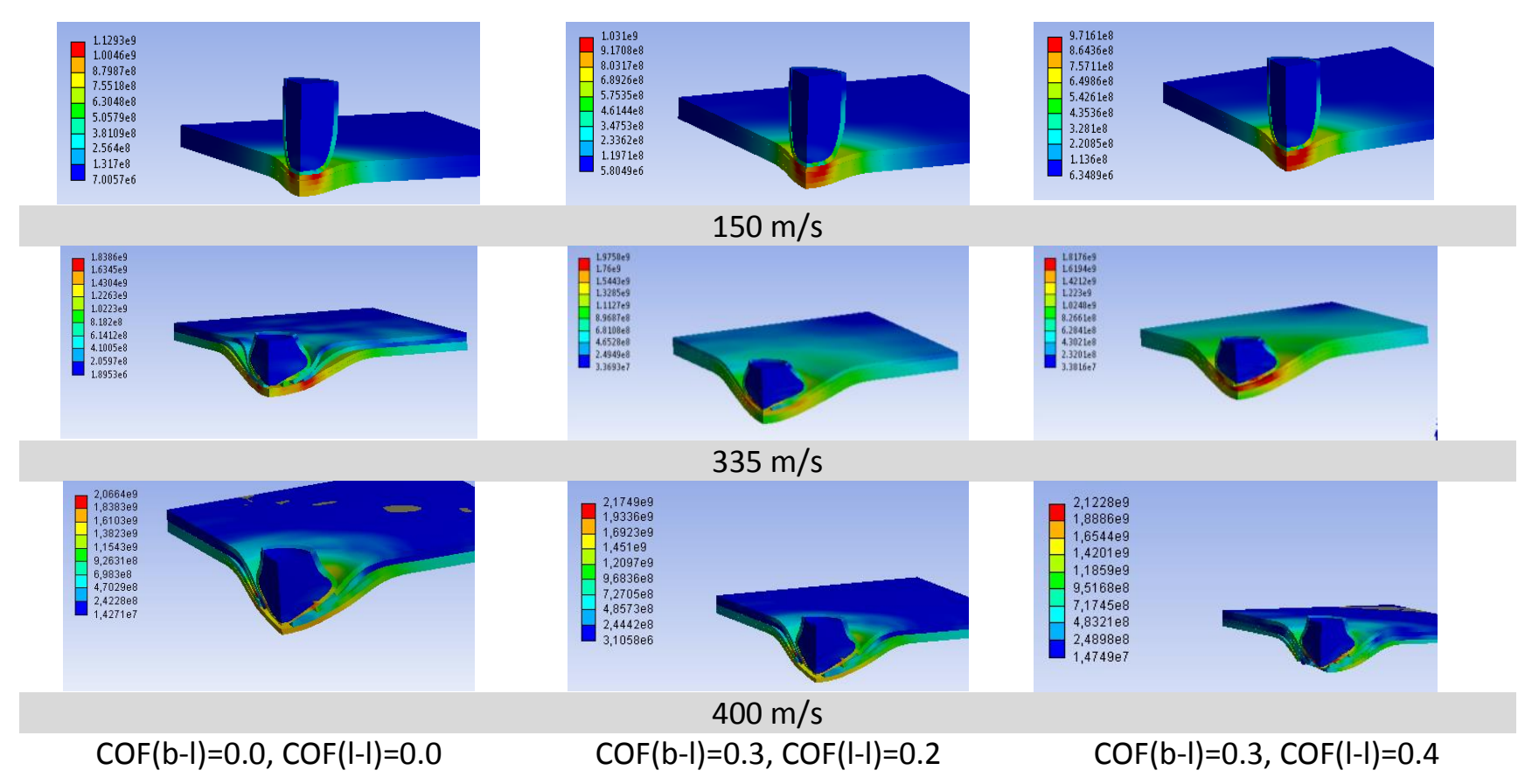

Figure 14. Influence of friction coefficient, for the same moment of simulation, $t=3 \times 10^{-5} \mathrm{~s}$

The validation could imply the number of broken layers, the residual velocity (that could be measured), the backface signature, the number of broken yarns etc.

Grujicic et al. [34] used a simple penaltybased algorithm for modeling yarn/yarn and projectile/fabric interactions. A Coulomb model was for friction as a viscous model of friction was not yet supported by the dedicated soft. Two frictional cases were considered: a) both the yarn/yarn friction coefficient $\mu_{\mathrm{y} / \mathrm{y}}$ and the projectile/fabric friction coefficient $\mu_{\mathrm{p} / \mathrm{f}}$ are set to 0.5 , and $\mathrm{b}$ ) no friction, meaning $\mu_{\mathrm{y} / \mathrm{y}}=\mu_{\mathrm{p} / \mathrm{f}}=0$. Boundary conditions consider all four fabric edges are fixed. The aramid yarn (Kevlar 129) has orthotropic linear elastic characteristics, Examples of the temporal evolution of deformation within the fabric obtained using the yarn-level FEM analysis and the unit-cell based FEM analysis are displayed in Fig. 15.

For the no-friction case, yarns were substantially displaced in the in-plane directions, away from the center of impact.

Thus, the friction at the yarn crossovers provides resistance to the relative tangential motion of the yarns, while such resistance is absent in the no-friction case. In the nofriction case, yarns impacted by the projectile are pushed outward, a fewer number of yarns are broken and the projectile manages to penetrate the fabric mainly by "wedging" through it.
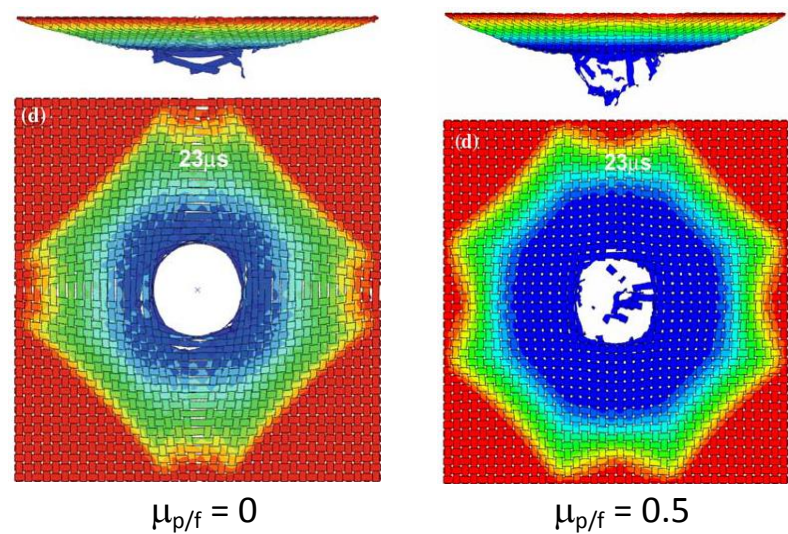

Figure 15. The deformation in the fabric for the yarn-level FEM model Contour bands correspond to different values of the displacements normal to the fabric surface [35]

Ojoc et al. [35] simulated the behavior of unidirectional fabrics, with isotropic yarns (similar to aramid ones) under the impact of a $9 \mathrm{~mm}$ bullet. The yarns of a layer were perpendicularly to the direction of yarns in the previous layer $\left(0^{\circ}, 90^{\circ}, 0^{\circ}, 90^{\circ}\right)$. The friction coefficient was constant, $\mu_{\text {projectile-yarn }}=0.3$ and $\mu_{\text {projectile-yarn }}=0.4$. For all these cases, the impact velocity was varied between 100 and 400 $[\mathrm{m} / \mathrm{s}]$ with an increment of $100 \mathrm{~m} / \mathrm{s}$, in order to understand how the panel is damaged. 


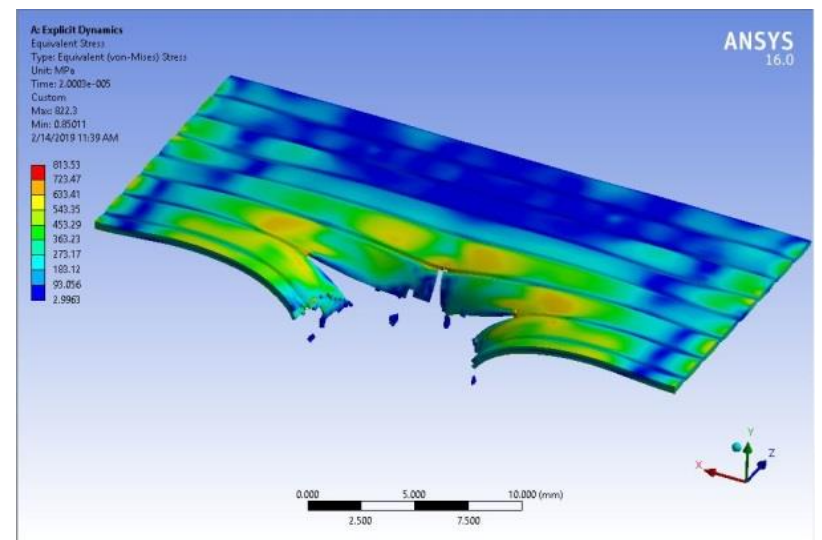

Layer 1

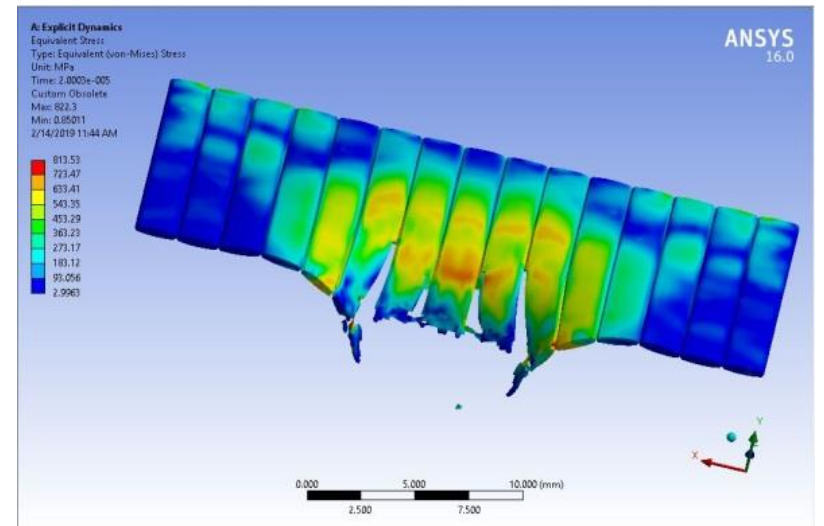

Layer 2

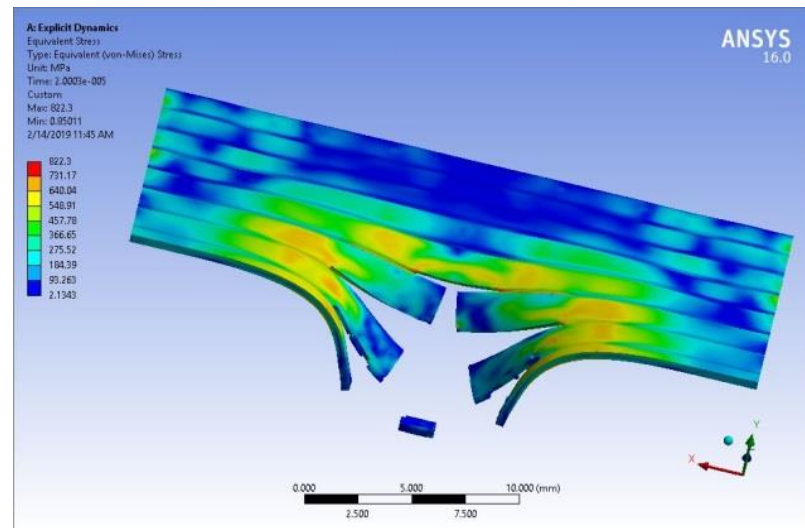

Layer 3

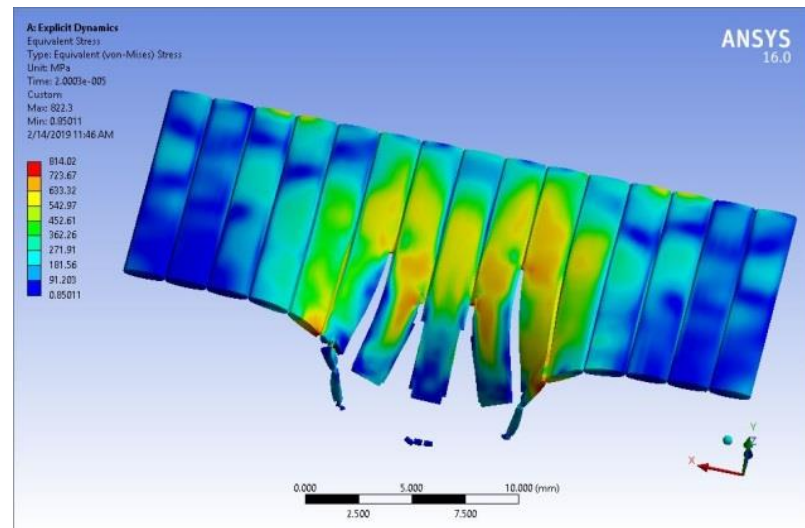

Layer 4

Figure 16. Impact simulated on four layers made of unidirectional yrans, at moment $\mathrm{t}=2 \times 10^{-5} \mathrm{~s}$ impact velocity $400 \mathrm{~m} / \mathrm{m}$, the bullet being transparent [34]
For the four layer package, for lower velocities, the residual velocity has a lower percentage of the impact velocity, but at high speeds (Fig. 16), for this package, the reduction of the residual velocity from the impact velocity is even lower $\left(2.7 \%\right.$ at $\mathrm{v}_{0}=300$ $\mathrm{m} / \mathrm{s}$ and only $1.75 \%$ at $\mathrm{v}_{0}=400 \mathrm{~m} / \mathrm{s}$ ).

In the absence of friction the projectile can wedge through the fabric, while in the presence of friction more yarn failure is required before the projectile can reach its residual velocity.

Nilakantan et al. [36], [37] simulated the impact of a fabric, using a static frictional coefficient of 0.18 for the yarn to yarn contact and 0.18 for the projectile to yarn contact algorithms. The thickness of the warp and fill yarns is $0.115 \mathrm{~mm}$ and the total fabric thickness at the cross-over locations is 0.23 $\mathrm{mm}$. The yarn count in both warp and fill directions is 34 yarns per inch. Two impact velocities of $100 \mathrm{~m} / \mathrm{s}$ and $200 \mathrm{~m} / \mathrm{s}$ are selected. While the projectile is penetrating through a hole in the fabric which is smaller than the projectile's diameter, the frictional energy dissipated by the projectile-yarn interaction rises to its maximum level. While the fabric is springing back after complete projectile penetration, the frictional energy dissipated by yarn-yarn sliding interactions rises to its maximum level.

\section{CONCLUSION}

Recent works simulating the impact projectile - target, done at different levels (micro, meso, macro) pointed out the influence of friction coefficient between bodies in contact. In all cited references, the friction coefficient is kept constant, even if experimental studies proved that it has different values for different sliding speeds, especially for contact metal - polymer as in the impact of aramid fabric armor.

\section{ACKNOWLEDGEMENT}

This paper was supported by a grant of the Romanian Ministery of Research and 
Innovation, CCCDI-UEFISCDI, project no. PN-IIIP1-1.2-PCCCDI-2017-0404/31PCCDI/2018, within PNCDI III and by Doctorale School for Mechanical and Industrial Engineering of "Dunarea de Jos" University of Galati.

\section{REFERENCES}

[1] N.K. Myshkin, M.I. Petrokovets, A.V. Koval: Tribology of polymers: Adhesion, friction, wear, and mass-transfer, Tribology International, Vol. 38, pp. 910-921, 2005.

[2] K. Shooter, R.H. Thomas: Frictional properties of some plastics. Research, Vol. 2, pp. 533539, 1952.

[3] T. Fort: Adsorption and boundary friction of polymer surfaces, Journal of Physical Chemistry, Vol. 66, pp. 1136-1143, 1962.

[4] N.S. White: Small oil-free bearings. Journal of research of the National Institute of Standards and Technology, Vol. 57, pp. 185-189, 1956.

[5] G.M. Bartenev, V.V. Lavrentev: Friction and wear of polymers, Elsevier; p. 212, Amsterdam, 1981.

[6] D.G. Flom, N.T. Porile: Effect of temperature and hight-speed sliding on the friction of teflon on teflon, Nature, Vol. 175, pp. 682-685, 1955.

[7] G. W. Stachowiak, A.W. Batchelor: Engineering tribology, ButterworthHeinemann, Team Lrn., 2005.

[8] C.M. McC. Ettles: Polymer and Elastomer Friction in the Thermal Control Regime, ASLE Transactions, Vol. 30, pp. 149-159, 1987

[9] A.M. Bueche, D.G. Flom: Surface Friction and Dynamic Mechanical Properties of Polymers, Wear, Vol. 2, pp. 168-182, 1958/1959.

[10]G. Nilakantan, R. L. Merrill, M. Keefe, J.W. Gillespie Jr., E.D. Wetzel: Experimental investigation of the role of frictional yarn pullout and windowing on the probabilistic impact response of Kevlar fabrics, Composites: Part B Vol. 68, pp. 215-229, 2015.

[11]Opportunities in Protection Materials Science and Technology for Future Army Applications, ISBN 978-0-309-21285-4, 2011.

[12] S. Sockalingam, S. C. Chowdhury, J. W. Gillespie Jr., M. Keefe: Recent advances in modeling and experiments of Kevlar ballistic fibrils, fibers, yarns and flexible woven textile fabrics - a review, Textile Research Journal, Vol. 87, No. 8, pp. 984-1010, 2017.

[13] D. Prevorsek: 'Spectra: The lastest entry in the field of high-performance fibers., in Lewin, M. and Preston, J. (eds) Handbook of Fiber Science and Technology, High Technology Fibers Part D, Marcel Dekker, Vol. 3, p. 17, 1996.

[14] Q.P. McAllister, J.W. Gillespie Jr., M.R. Van Landingham: The influence of surface microstructure on the scratch characteristics of Kevlar fibers, Journal of Materials Science; Vol. 48: pp. 1292-1302, 2013.

[15] S. Sockalingam, J. W Gillespie Jr, M. Keefe: Influence of multiaxial loading on the failure of Kevlar KM2 single fiber, Textile Research Journal, Vol. 88,No. 5, pp. 483-498, 2018.

[16] M. Sava, A. Hadăr, I. Părăuşanu, H.A. Petrescu, F. Baciu, S.M. Marinel: Validation of the numerical model of single-layer composites reinforced with carbon fiber and aramid, AIP Conference Proceedings 1738 (1), 480080.

[17] A. Bhatnagar: Lightweight ballistic composites, CRC Press, Boca Raton Boston New York, 2006.

[18] S. Rebouillat: Tribological properties of woven para-aramid fabrics and their constituent yarns, Journal of Material Science, Vol. 33, pp. 3293-3301, 1998.

[19] C.T. Lim, V.B.C. Tan, C.H. Cheong: Perforation of high-strength double-ply fabric system by varying shaped projectiles. International Journal of Impact Engineering, Vol. 27, No. 6, pp. 577-591, 2002.

[20] V. B. C. Tan, K. J. L. Khoo: 'Perforation of flexible laminates by projectiles of different geometry,' International Journal of Impact Engineering, Vol. 31, pp. 793-810, 2005.

[21] P. M. Cunniff: 'An Analysis of the System Effects in Woven Fabric Under Ballistic Impact, Textile Research Journal, Vol. 62, No. 9, pp. 495-509, 1992.

[22] L. Lee, S. Rudov-Clark, A.P. Mouritz, M.K. Bannister, I. Herszberg: 'Effect of weaving damage on the tensile properties of threedimensional woven composites,' Composite Structures, Vol. 57, pp. 405-413, 2002.

[23] S. Rudov-Clark, A.P. Mouritz, L. Lee, M. K. Bannister 'Fibre damage in the manufacture of advanced three-dimensional woven composites,' Composites: Part A, Vol. 34, pp. 963-970, 2003.

[24] C.T. Lim, V.P.W. Shim, Y.H. Ng: Finite-element modeling of the ballistic impact of fabric armor, International Journal of Impact Engineering, Vol. 28, pp. 13-31, 2003.

[25] Ballistic material handbook, Teijin, available at: https://www.teijinaramid.com/wpcontent/uploads/2018/03/TEIJ_Handbook_Balli stics_2018_WEB.pdf, accessed at 21.04.2019. 
[26] C. Pirvu: Contributions on numerical and experimental study of ballistic packages made of aramid fibers (in Romanian) PhD thesis, Dunarea de Jos University, 2015.

[27] S. Sockalingam, J.W. Gillespie Jr., M. Keefe: On the transverse compression response of Kevlar KM2 using fiber-level finite element model, International Journal of Solids and Structures, Vol. 51, pp. 2504-2517, 2014.

[28] H.L. Thomas: 'Needle-Punched Non-Woven Fabric for Fragmentation Protection', 14th International Conference on Composite Materials, Society of Manufacturing Engineers, July 14-18, 2003.

[29] Y. Duan, M. Keefe, T.A. Bogetti, B.A. Cheeseman: 'Modeling friction effects on the ballistic impact behavior of a single-ply high-strength fabric, International Journal of Impact Engineering, Vol. 31, No. 8, pp. 996-1012, 2005.

[30] Y. Wang, Y. Miao, L. Huang, D. Swenson, C.-F. Yen, J. Yu, J. Q. Zheng: Effect of the inter-fiber friction on fiber damage propagation and ballistic limit of 2-D woven fabrics under a fully confined boundary condition, International Journal of Impact Engineering, Vol. 97, pp. 66-78, 2016.

[31] Y. Duan, M. Keefe, T.A. Bogetti, B.A. Cheeseman: 'Modeling the role of friction during ballistic impact of a high-strength plainweave fabric,' Composite Structures, Vol. 68, No. 3, pp. 331-337, 2005.

[32] A. Wilde, D. Roylance, J.M. Rogers: 'Photographic investigation of high-speed missile impact upon nylon fabric, Part 1: Energy absorption and cone radial velocity in fabric,' Textile Research Journal, Vol. 12, pp. 753-761, 1973.

[33] T.F. Ionescu, C. Pirvu, S. Badea, C. Georgescu, L. Deleanu: The Influence of Friction Characteristics in Simulating the Impact Bullet - Stratified Materials, 15th International Conference on Tribology, Kragujevac, Serbia, 17 - 19 May 2017.

[34] W.C.M. Grujicic, B. AE T. He, B. A. Cheeseman: Development and verification of a meso-scale based dynamic material model for plainwoven single-ply ballistic fabric J Mater Sci, Vol. 43, pp. 6301-6323, 2008.

[35] G. G. Ojoc, L. Oancea, Catalin Pirvu, S. Sandu, L. Deleanu, Modeling of Impact on Multiple Layers with Unidirectional Yarns, Scientific Conference of Doctoral Schools, Dunarea de Jos University of Galati, 2019.

[36] G. Nilakantan, R. L. Merrill, M. Keefe, J.W. Gillespie Jr., E.D. Wetzel: Experimental investigation of the role of frictional yarn pullout and windowing on the probabilistic impact response of kevlar fabrics, Composites: Part B Vol. 68, pp. 215-229, 2015.

[37] G. Nilakantn, M. Keefe, T. A. Bogetti, J.W. Gillespie Jr.: Multiscale modeling of the impact of textile fabrics based on hybrid element analysis, International Journal of Impact Engineering , Vol. 37, pp. 1056-1071, 2010. 